\title{
Efecto de la temperatura en la estructura y morfología de recubrimientos de $(\mathrm{Ti}, \mathrm{Al}) \mathrm{N}$
}

\author{
Mónica Emperatriz Bernal Salamanca ${ }^{\star}$, Vicente Javier Benavides Palacios ${ }^{\star \star}$,

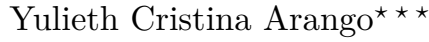 \\ Universidad Nacional de Colombia sede Manizales. Manizales - Colombia
}

FECHA DE ENTREGA: 5 DE MARZO DE 2012

FECHA DE APRobACIÓN: 9 DE OCTUBRE DE 2012

\begin{abstract}
Resumen Películas del (Ti,Al)N fueron crecidas sobre un sustrato de acero inoxidable por la técnica PAPVD por arco pulsado controlado usando un blanco de $\mathrm{TiAl}$ (50/50\% atómico) con atmósfera $\mathrm{Ar}+\mathrm{N}_{2}$. Se crecieron a diferentes temperaturas del sustrato, a temperatura ambiente, $50{ }^{\circ} \mathrm{C}$ y $100{ }^{\circ} \mathrm{C}$. Los recubrimientos fueron caracterizados estructuralmente con difracción de rayos $\mathrm{X}$ (XRD), con el fin de determinar las microtensiones, el tamaño del cristalito y el parámetro de red; en función de la temperatura del sustrato se utilizó el método Rietveld con funciones de ajuste PVII y SPV. La caracterización morfológica se realizó con la técnica de microscopia de fuerza atómica (AFM), obteniendo resultados de rugosidad y tamaño de grano. Los resultados muestran que la orientación (200) es el plano de mayor intensidad que se mantiene para las diferentes temperaturas. La rugosidad de la película decrece a medida que la temperatura del sustrato aumenta.
\end{abstract}

Abstract (Ti,Al)N films were grown on a susbtrate of stainless steel by means PAPVD by controlled pulsed cathodic arc technique using a target of $\mathrm{TiAl}$ (50/50\% atomic) in an $\mathrm{Ar}+\mathrm{N}_{2}$ atmosphere. The coatings were deposited at different temperatures of the substrate, room temperature, $50{ }^{\circ} \mathrm{C}$ and $100{ }^{\circ} \mathrm{C}$. The films were characterized structurally by means X-ray diffraction (XRD). With the purpose of determining microstrain, crystallite size and lattice parameter in function of the substrate temperature was used Rietveld method with PVII and SPV fitting funtions. The morphology study was made by means of atomic force microscopy (AFM), obtaining results about roughness and grain size. The results show that (200) orientation has more intensity than other planes and it keeps for different temperatures. The roughness diminishes with the increment of the substrate temperature.

Palabras Clave: (Ti,Al)N, análisis estructural, morfología de la superficie.

Keywords: (Ti,Al)N, structural analysis, surface morphology.

\footnotetext{
* mebernals@unal.edu.co

** vbenavidez@gmasltda.com

$\star \star \star$ ycarango@unalmzl.edu.co
} 


\section{Introducción}

Las películas delgadas de $(\mathrm{Ti}, \mathrm{Al}) \mathrm{N}$ crecidas por deposición física, en fase vapor (PVD), se han incrementado rápidamente sus aplicaciones industriales por sus grandes propiedades mecánicas y tribológicas y alta resistencia a la temperatura de oxidación [1. Tales recubrimientos han sido crecidos por diferentes técnicas de PVD, como sputtering por magnetrón y evaporación por arco catódico [2] La incorporación de aluminio en la estructura cúbica fcc de TiN lleva a mejorar la estabilidad térmica del recubrimiento. Esta sustitución no cambia el ordenamiento de la estructura cristalina cúbica centrada en las caras, pero suministra una solución sólida fuerte que resulta en una alta dureza asociada a un incremento en la resistencia al desgaste. Las sustituciones atómicas en la que los átomos de titanio son parcialmente sustituidos por los átomos de aluminio pueden dar una alta estabilidad química y mejorar la resistencia a la oxidación (es decir, los átomos de aluminio son menores que los de titanio) [3]. En el presente trabajo, se crecieron películas $(\mathrm{Ti}, \mathrm{Al}) \mathrm{N}$ con la técnica PAPVD por arco catódico, para analizar el efecto de la temperatura del sustrato en la micro-estructura del recubrimiento.

\section{Detalle experimental}

Las películas delgadas de $(\mathrm{Ti}, \mathrm{Al}) \mathrm{N}$ fueron crecidas en sistema no comercial de arco pulsado controlado que utiliza tecnología IGBT [4]. Los recubrimientos fueron depositados sobre un sustrato de acero inoxidable a una presión de trabajo constante de 3.5 mbar $\left(\mathrm{Ar}+\mathrm{N}_{2}, 10 \%+90 \%\right)$, un potencial de $270 \mathrm{~V}$ y una distancia entre ánodo y cátodo de $5 \mathrm{~mm}$. El blanco utilizado es de TiAl con composición atómica de 50/50\%. Las películas se crecieron a tres temperaturas del sustrato diferente: temperatura ambiente $20{ }^{\circ} \mathrm{C}, 50{ }^{\circ} \mathrm{C}$ y $100{ }^{\circ} \mathrm{C}$. El análisis estructural se realizó con un XRD Bruker AXS modelo D8 Advance, geometría theta/theta, radiación $\mathrm{CuK} \alpha(\lambda=1.5406 \AA, V=40 \mathrm{kV}, I=30 \mathrm{~mA})$ en el modo de incidencia razante. La morfología superficial se realizó con un microscopio de barrido por sonda (SPM) de Park Scientific Instruments, modelo AutoProbe CP ,en el modo de microscopía de fuerza atómica (AFM).

\section{Resultados y discusión}

Las películas de $(\mathrm{Ti}, \mathrm{Al}) \mathrm{N}$ crecieron con una estructura cristalina cúbica FCC con grupo espacial fm-3m (figura 1). Esta estructura cristalina es similar a la estructura del compuesto TiN pero con sustituciones de átomos de titanio por átomos de aluminio, ocasionando un incremento en la posición 2 theta de los picos con respecto a TiN. Este corrimiento se debe al incremento de la fase AlN en la red [5]. (Ti,Al)N se puede cristalizar en una estructura hexagonal dependiendo de la concentración de aluminio en la red, cuando la concentración de aluminio esta por debajo de $x=0.4$ en $\mathrm{Ti}_{1-x} \mathrm{Al}_{x} \mathrm{~N}$ se forman fases hexagonales como $\mathrm{Ti}_{3} \mathrm{Al}_{2} \mathrm{~N}_{2}$ y $\mathrm{Ti}_{2} \mathrm{AlN}$. Estas estructuras no se evidenciaron en los difractogramas 


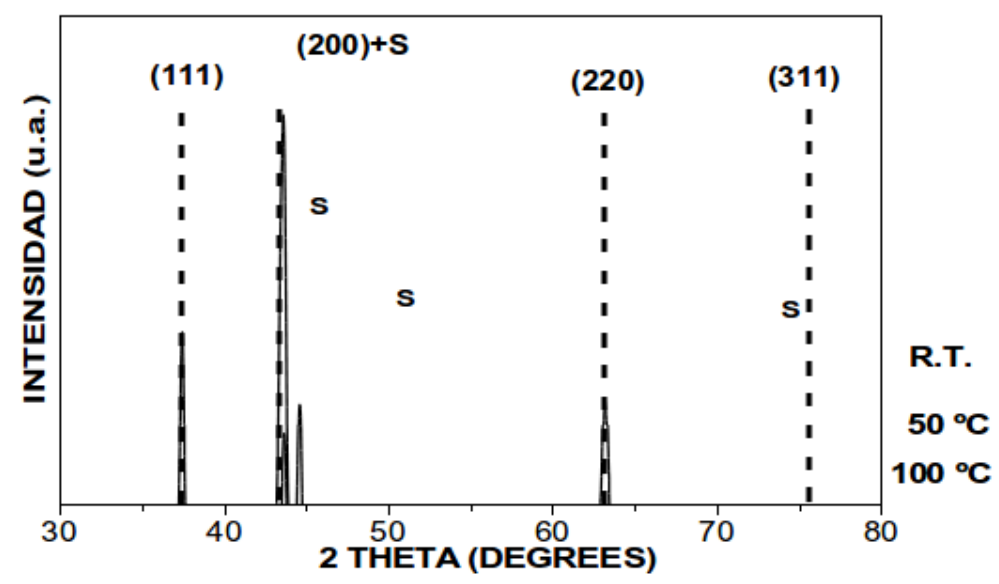

Figura 1. Patrones $\mathrm{XRD}$ de $(\mathrm{Ti}, \mathrm{Al}) \mathrm{N}$ variando la temperatura del sustrato.

porque las películas crecidas presentan una concentración de aluminio por encima de $x=0.4$ [6]. La elevada concentración de aluminio en las películas puede ser explicada desde el punto de vista de la descarga por arco. El aluminio es un material con bajo punto de fusión y buena conductividad eléctrica por lo tanto, los spots catódicos fundirán con mayor facilidad el aluminio ocasionando una emisión explosiva con grandes concentraciones de especies iónicas de aluminio [7]. Los refinamientos de los difractogramas fueron hechos mediante el uso del método Rietveld, con el objetivo de obtener el FWHM de los picos, observando que el pico (200) de (Ti,Al)N se superpone con el pico (110) de hierro que pertenece al sustrato. Para tal fin, se utilizaron dos funciones de refinamiento PVII (Pearson 7) y SPV (Split Pseudo Voigt). En los refinamientos se consideró el factor de polarización de Lorentz (26.37) para el monocromador de grafito en el plano secundario y un ensanchamiento instrumental de $0.003514^{\circ}$ [8]. El tamaño del cristalito $(\mathbf{D})$ y la microdeformación $(\varepsilon)$ fueron determinados utilizando estas dos funciones (Cuadro 1). A $50{ }^{\circ} \mathrm{C}$, se observa una reducción del tamaño de cristalito, cuando hay incrementos de temperatura se ha podido explicar como procesos de difusión que interrumpen el crecimiento de los granos; en (Ti,Al)N difusión de $\mathrm{AlN}$ y $\mathrm{Al}_{2} \mathrm{O}_{3}$ puede aparecer. El óxido de aluminio siempre está presente en la película y es una de las causas de la elevada resistencia de $(\mathrm{Ti}, \mathrm{Al}) \mathrm{N}$ a la oxidación 9].

El cuadro 1 no muestra cambios considerables en el parámetro de red como función de la temperatura. Los incrementos en la microdeformación de las películas se pueden asociar a dislocaciones en la red y fronteras de grano a bajos ángulos principalmente [10]. Con las dos funciones de ajuste se obtuvieron valores muy 


\begin{tabular}{|c|c|c|c|c|c|c|}
\hline \multirow{3}{*}{$\frac{\text { Temperatura }\left({ }^{\circ} \mathbf{C}\right)}{\text { Parámetro de red }(\AA)}$} & \multicolumn{2}{|c|}{ R.T. } & \multicolumn{2}{|c|}{$50{ }^{\circ} \mathrm{C}$} & \multicolumn{2}{|c|}{$100{ }^{\circ} \mathrm{C}$} \\
\hline & PVII & SPV & PVII & SPV & PVII & SPV \\
\hline & 4.169 & - & 4.168 & - & 4.167 & - \\
\hline $\mathbf{D}(\mathrm{nm})$ & 64.4 & 66.5 & 43.3 & 41 & 76.5 & 76.6 \\
\hline$\varepsilon(\operatorname{adim}) \times 10^{-3}$ & 285 & 279 & 296 & 292 & 335 & 343 \\
\hline $\mathbf{D}(\mathrm{nm})$ promedio & \multicolumn{2}{|c|}{$65.45 \pm 1.05$} & \multicolumn{2}{|c|}{$42,15 \pm 1.15$} & \multicolumn{2}{|c|}{$76.55 \pm 0.05$} \\
\hline$\varepsilon(\operatorname{adim}) \times 10^{-3}$ promedio & \multicolumn{2}{|c|}{$282 \pm 3$} & \multicolumn{2}{|c|}{$294 \pm 2$} & \multicolumn{2}{|c|}{$339 \pm 4$} \\
\hline
\end{tabular}

Cuadro 1. Cálculo de Parámetros Estructurales, utilizando dos funciones de ajuste.

aproximados de $\mathbf{D}$ y $\varepsilon$ con los cuales se encontró una posibilidad para evaluar con una mejor precisión estos parámetros.

Morfológicamente las muestras de $(\mathrm{Ti}, \mathrm{Al}) \mathrm{N}$ se estudiaron con SPM, en el modo de microscopia de fuerza atómica en contacto (AFM-C). La figura 2 muestra imágenes AFM en un área de barrido de $5 \mu \mathrm{m}^{2}$ a temperaturas de (a) ambiente, (b) $50{ }^{\circ} \mathrm{C} \mathrm{y} \mathrm{(c)} 100{ }^{\circ} \mathrm{C}$.

Al utilizar esta técnica se observa cómo la temperatura del sustrato afecta la morfología de la película; pues para esta película depositada a temperatura ambiente $\left(20^{\circ} \mathrm{C}\right)$, la superficie es muy irregular mientras que la superficie de la película depositada a una temperatura de $100{ }^{\circ} \mathrm{C}$, su detalle morfológico es mucho más suave. Esto demuestra que la temperatura favorece la coalescencia del material depositado.

En la Figura 2a, se observa una superficie demasiado irregular, donde muestra un proceso continuado de nucleación de granos, debido a la baja movilidad de los adatomos. Las Figuras 2b y 2c, muestran una superficie más suave debido a una mayor movilidad de los adatomos que favorecen la coalescencia. Este comportamiento se puede apreciar mucho mejor en la Figura 3 (parte inferior), el cual muestra la dependencia de la rugosidad raíz cuadrática media (Rms) con la temperatura del sustrato. La rugosidad Rms disminuye con la temperatura del sustrato. Un incremento en la temperatura de deposición lleva a un perfeccionamiento de la movilidad superficial de los adatomos, así como a la difusión atómica.

El tamaño del grano se mantiene relativamente constante, entre $254 \pm 42$ y $270 \pm 38 \mathrm{~nm}$, a pesar del cambio de temperatura en el sustrato, esto sugiere que dicho intervalo de temperatura no es suficiente para que este parámetro contribuya en el crecimiento de los granos del recubrimiento, tal como lo reportan varios autores que han escrito acerca de películas de TiN [10]. 


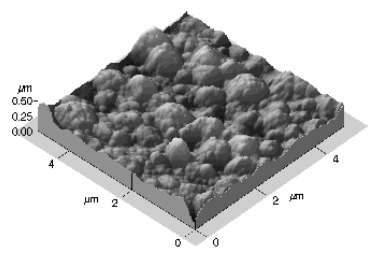

(a)

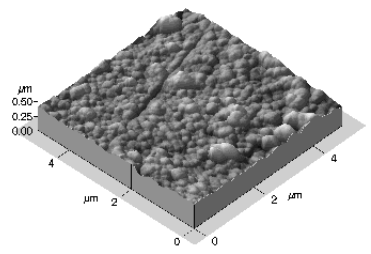

(b)

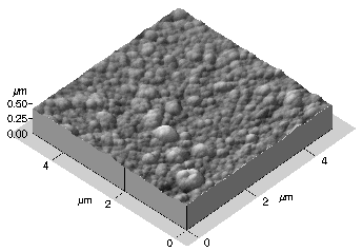

(c)

Figura 2. Imagen AFM de $5 \mu \mathrm{m}^{2} \mathrm{de}(\mathrm{Ti}, \mathrm{Al}) \mathrm{N}$ a (a) R.T., (b) $50{ }^{\circ} \mathrm{C}$ y (c) $100{ }^{\circ} \mathrm{C}$.

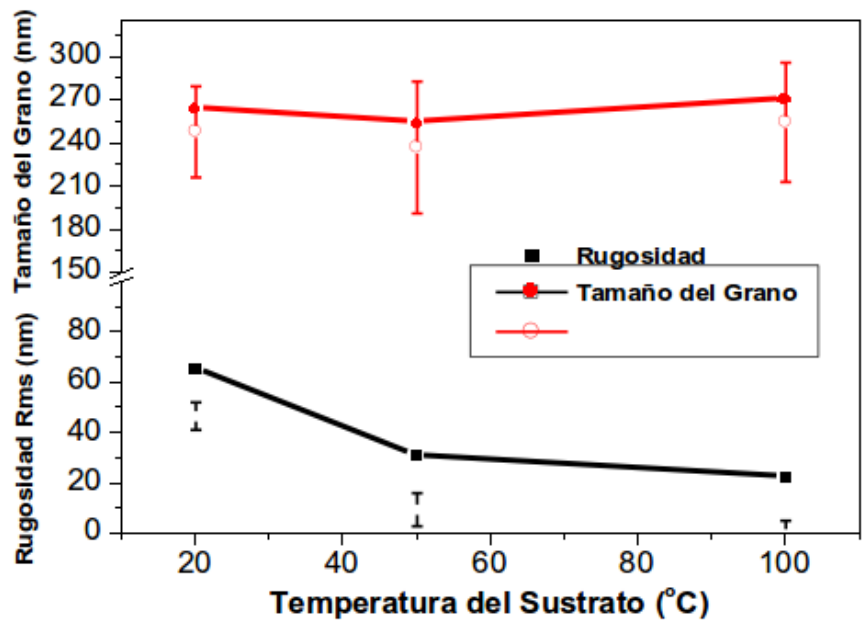

Figura 3. Evolución del tamaño del grano (curva superior) y rugosidad Rms en función de la temperatura.

\section{Conclusiones}

Las películas de $(\mathrm{Ti}, \mathrm{Al}) \mathrm{N}$ presentaron un incremento en la microdeformación con el aumento de la temperatura del sustrato, mientras que el tamaño del cristalito no presenta una tendencia. Estos dos parámetros fueron evaluados con dos funciones de ajuste, usando el método Rietveld. La temperatura del sustrato favorece la disminución de la rugosidad de la película, y frente al tamaño del grano su valor es constante.

\section{Referencias}

1. Paldey, S., Deevi, S.C.: Mater. Sci. Eng., A 342, 58. (2003)

2. Weber, F., Fontaine, F., Scheib, M., Bock, W.: Surf. Coat. Technol. 177-178, 227. (2004) 
3. Prengel, H.G., Santhanam, A.T., Penich, R.M., Jindal, P.C., Wendt, K.H.: Surf. Coat. Technol. 94-95, 597. (1997)

4. Jiménez, H., Devia, D.M., Benavides, V., Devia, A., Arango Y.C., Velez, J.M.: Materials Characterization, In Press, Accepted Manuscript, Available online 4 September 2007. (2007)

5. Wahlström, U., Hultman, L., Sundgren, J-E., Adibi, F.: Thin Solid Films 235, 62. (1993)

6. Hultman, L.: Vacuum. 57, 1. (2000)

7. Martin, P.J., McKenzie, D.R.: Handbook Vacuum Arc Science and Technol. edit. by R. Boxman. (1995)

8. Manual de Usuario. Diffrac Plus, Bruker-AXS Topas 3 (2005).

9. McIntyre, D., Greene, J., Hakansson, G., Sundgren, J-E., Münz, W.: J. Appl Phys 67, 1542. (1990)

10. Cheng, Y.H., Tay, B.K., Lau, S.P.: J. Vac. Sci. Technol. A 20(4) Jul/Aug 2002 pag. 1270. (2002) 